\title{
Condutas antissociais e delitivas e habilidades sociais em contexto forense ${ }^{1}$
}

\author{
Antisocial, criminal behaviors and social skills in forensic context
}

\author{
Catarina Isabel Fernandes Sintra ${ }^{[a]}$, Paulo Lopes ${ }^{[b]}$, Nilton Formiga ${ }^{[c]}$
}

[a] Psicóloga criminal e do comportamento desviante pela Universidade Lusófona de Humanidades e Tecnologias de Lisboa, técnica na Junta de Freguesia do Socorro, Lisboa - Portugal, e-mail: cathy.sintra@gmail.com

[b] Psicólogo clínico pela Universidade Lusófona de Humanidades e Tecnologias de Lisboa, Doutorando na Universidade de Salamanca, docente universitário, Lisboa - Portugal, e-mail: pjflopes@gmail.com

[c] Mestre em Psicologia Social pela Universidade Federal da Paraíba, Doutorando na mesma universidade, João Pessoa, PB - Brasil, e-mail: nsformiga@yahoo.com

\section{Resumo}

O problema do consumo de drogas continua a ser um tema social complexo, alvo privilegiado de curiosidade e de preocupação social, política e científica. Hoje, mais do que nunca, a toxicodependência surge na encruzilhada de múltiplas dimensões inclusa na via humana, designando, na maioria das vezes, uma representação de usos nocivos, autodestrutivos e alienantes. Nesse sentido, a presente investigação teve como objectivo estudar a relação entre o consumo de drogas ilícitas e as condutas antissociais e delitivas/delinquentes em toxicodependentes. A investigação foi de natureza transversal, constituída por uma amostra de 124 sujeitos de ambos os sexos, com uma média de idades de 31,45 (DP = 7,58). Os resultados do estudo demonstraram que os toxicodependentes apresentam índices mais elevados de condutas antissociais, bem como de condutas delitivas, comparativamente ao grupo de controlo. Verificou-se também a existência de diferenças no nível das habilidades sociais entre os grupos.

Palavras-chave: Condutas antissociais e delitivas. Habilidades sociais. Agressividade e toxicodependência.

1 No presente artigo, foi mantida a grafia do país de origem. 


\begin{abstract}
The problem of drug use remains a complex social issue, prime target of social, political and scientific curiosity and concern. Today, more than ever, addiction arises at the crossroads of multiple dimensions included in the human pathway. Most of the time, it characterizes a harmful, self-destructive and alienating representation. This research aimed at studying the relation between the consumption of illicit drugs and antisocial, criminal conducts among drug addicts. The examination had a cross-cutting nature, consisting of a sample of 124 subjects of both sexes, with an average age of 31.45 (SD =7.58). The results showed that drug users have higher rates of antisocial conduct, as well as criminal conduct, compared with the control group. It was also noted that there are differences in the level of social skills between the groups.
\end{abstract}

Keywords: Antisocial and criminal behaviors. Social skills. Hostility and drug addiction.

\section{Introduçáo}

Actualmente em Portugal o consumo de drogas é, à semelhança de outros países, um tema social complexo, alvo privilegiado de curiosidade e de preocupação social, política e científica (Quintas, 2000). Hoje, mais do que nunca, a toxicodependência surge na encruzilhada de múltiplas dimensões que se interpenetram na via humana, designando na maioria das vezes a figura caricatural de usos nocivos, autodestrutivos e alienantes (Morel, Hervé \& Fontaine, 1998).

O fenómeno de uso de drogas não é recente, existe há muitos anos enraizado em praticamente toda a história das sociedades. Porém, nas últimas três décadas, assistiu-se ao aparecimento de novas drogas, novas formas de consumo e ao aumento significativo da variedade e número de consumidores (Ferros, 2003). Este facto leva-nos a questionar o que leva as pessoas a este sacrifício, ou seja, por que consomem? Segundo o Instituto da Droga e da Toxicodependência (2007), os sujeitos que decidem consumir uma droga estão a fazer uma opção, uma escolha, sendo esta influenciada por inúmeros factores. Torna-se assim fundamental efectuar pesquisas sobre os factores preditivos do abuso de drogas, ou seja, é importante perceber quais são os factores de risco (que levam à experimentação e ao uso continuado) e quais os factores protectores (que inibem a experimentação) que existem ou podem ser desenvolvidos, uma vez que o desenvolvimento humano saudável resulta de um equilíbrio entre os factores de protecção e de risco, determinado não apenas na contabilização do número de variáveis de um e de outro tipo, mas sobretudo na sua frequência respectiva, duração, severidade e etapa do desenvolvimento em que ocorrem (Abraão, 1999).

Neste âmbito, surgiram diversas investigações. Patrício (1996) destaca, por um lado, as "grandes" descobertas de factores de âmbito individual, psicológico, físico e social (que ocorrem significativamente na adolescência) que podem provocar um sofrimento que facilite a atitude de abertura aos consumos, e por outro, a curiosidade, a necessidade de ser aceite, de se integrar no grupo (medo da exclusão do grupo), de se afirmar diante dos seus semelhantes, de ser "diferente" ou sentir-se diferente e de chamar a atenção, o desejo de diversão, a disponibilidade da droga, a ilusão da resolução de problemas, uma representação positiva das substâncias, entre outros. A dominância destes factores é evidenciada num estudo realizado por Lopes e Faria (2005) com 254 toxicodependentes inseridos em programa de substituição opiácea de baixo limiar, no qual referem que os principais motivos apresentados para o uso de drogas são a curiosidade $(76,77 \%)$, os problemas pessoais $(9,84 \%)$, os problemas familiares $(9,45 \%)$, os problemas sociais $(2,36 \%)$ e outras causas $(1,57 \%)$, sendo a heroína $(51,18 \%)$ a droga mais consumida, seguindo-se a cocaína (26,38\%), o haxixe $(18,90 \%)$, alucinogéneos $(1,99 \%)$, benzodiazepinas/tranquilizantes $(0,79 \%)$ e as anfetaminas e cola $(0,79 \%)$.

Alguns investigadores mencionam que o problema da delinquência se encontra no cerne da pesquisa sobre a toxicodependência (Egger, Webb \& Reynolds, 1978). A questão do início da delinquência revela-se, desta forma, crucial. Tanto os trabalhos de Moffitt (1993) como os de Loeber (1990), 
tendem a corroborar que quanto mais precoces são as condutas delinquentes, maior é a probabilidade de que serão graves e duradouras. Logo, quanto mais antissocial é a criança, mais cedo se inicia na actividade delinquente, maior é o risco de vir a ser detida no futuro imediato, há uma maior duração do processo delituoso e há emergência de novas formas de comportamento antissocial.

Face a isto, a emergência do comportamento antissocial na infância deve ser alvo de uma intervenção precoce, se não, tende a persistir, e pode ser preditor de delinquência e de perturbações do comportamento mais graves, aumentando também o risco de abuso de substâncias na adolescência (Hawkins et al., 1992; Manger et al., 1992; Schulenberg et al., 1996; Tolan et al., 1995, citado por Abraão, 1999).

É importante ter em atenção que quando se procede à análise das condutas desviantes, estas devem ser compreendidas como um conjunto de condutas apresentadas pelos jovens no seu quotidiano, que se aglomeram em duas dimensões: as condutas antissociais e as condutas delitivas ou delinquentes (Formiga \& Gouveia, 2003): a conduta antissocial refere-se à não consciencialização das normas que devem ser respeitadas, ou seja, os sujeitos não expressam delitos, mas comportamentos que desafiam a ordem social e infringem as normas sociais; a conduta delitiva ou delinquente pode ser concebida como merecedora de punição, capaz de causar danos graves, morais e/ ou físicos (Molina \& Gomez, 1997), portanto, pode ser considerada mais severa que a antissocial, uma vez que representa uma ameaça eminente à ordem social vigente. Ambas as condutas interferem nos direitos e deveres das pessoas, ameaçando o seu bem-estar. $\mathrm{O}$ que as diferencia é a gravidade das suas consequências.

O estudo realizado por Brook, Whiteman, Balka e Cohen (1997) com adolescentes afro-americanos e porto-riquenhos, sobre as diferenças entre os factores de risco específicos para a delinquência e uso de drogas, revelou que ambas as fases, uso de droga e delinquência, reflectem factores comuns de domínios diversos - são eles: as interacções familiares precoces (e. g., fraca ligação recíproca entre pais e criança), as manifestações de personalidade (e. g., baixa integração do ego) e dimensões das atitudes escolares mais contemporâneas (e. g., tolerância de desvio e desvio de pares, baixa realização e realização de pares e ambiente escolar negativo).
Até o momento, relativamente à existência de uma relação entre a droga e a delinquência, sabe-se que não existe uma relação de causalidade simples entre as duas, e ainda menos, entre a droga e a criminalidade. Não deixa de ser verdade que um grande número de indivíduos detidos relatam o consumo de substâncias psicoactivas. Contudo, não se pode generalizar esta situação ao conjunto de todos os delinquentes - quanto muito, pode-se dizer que a droga e a delinquência, no sistema proibicionista que conhecemos, funcionam como factores de risco mútuos e que entre os grandes consumidores de cocaína e de heroína se instala geralmente uma ligação entre a droga e o crime.

Tal como refere Patrício (1996), um toxicodependente pode tornar-se secundariamente um delinquente e um marginal, bem como um delinquente se pode tornar um toxicodependente, ou seja, um doente. Assim, quem consome droga pode ser um doente, um delinquente, uma pessoa que está nas duas situações, ou uma pessoa que não está em nenhuma delas. A relação entre uma pessoa, um produto e um comportamento é, assim, complexa e não pode ser definida por um modelo conceptual linear. É fundamental lembrarmo-nos que nem todos os utilizadores de drogas se tornam dependentes - além do mais, para além da posse de drogas, nem todos se envolvem em actividades criminais, assim como os motivos que envolvem a delinquência podem diferir de indivíduo para indivíduo (Agra, 1997).

Relativamente ao uso de drogas e à agressividade, estudos realizados por Allen, Moeller, Rhoades e Cherek (1997), revelaram que existe uma maior agressividade em indivíduos com uma história de dependências de drogas do que numa população não consumidora, e que os sujeitos que usam drogas têm maior probabilidade de enveredar por comportamentos agressivos do que aqueles que não usam drogas (Muntaner, Walter, Nagoshi, Fishbein, Haertzen \& Jaffe, 1990). Este facto é corroborado por Loeber, (citado por Negreiros, 1998) que após efectuar uma revisão da literatura sobre a relação entre o consumo de drogas e a prática de comportamentos antissociais, também concluiu que os consumidores de drogas não apresentam, de um modo geral, comportamentos agressivos durante a infância, emergindo a agressividade concomitantemente com o abuso de drogas. Porém, ainda que várias drogas psicoactivas tenham demonstrado o aumento da agressividade 
sob condições experimentais (Bushman, 1993, citado por Allen et al. 1997), a associação entre a agressividade e o uso de drogas é mais complexa do que uma acção directa do abuso de droga(s) por si só causar agressividade num outro indivíduo não agressivo. Tal com refere Allen e cols. (1997), a agressividade pode ser uma resposta crescente à provocação, em vez de uma resposta forte ao uso de drogas.

Uma outra questão relativa ao consumo de drogas que se coloca é: existe alguma relação entre as habilidades sociais e a toxicodependência? Segundo Del Prette e Del Prette (2001), as habilidades sociais designam um conjunto de capacidades comportamentais aprendidas que envolvem interacções sociais. Estas correspondem a um universo abrangente das relações interpessoais e estendem-se para além da assertividade, incluindo as habilidades de comunicação, de resolução de problemas, de cooperação e as que são próprias dos rituais sociais estabelecidos pela subcultura grupal. Os mesmos autores afirmam que as habilidades sociais são reconhecidas como um factor de protecção no curso do desenvolvimento humano, pois têm sido associadas a melhor saúde física e mental, ao sucesso profissional e a maior realização pessoal.

Rubio e Anzano (1998), referem que as habilidades sociais se mostram como recursos indispensáveis para realizar actividades fundamentais da vida, como os relacionamentos interpessoais, a construção de amizades e o desenvolvimento de redes sociais, o trabalho em equipa e a prestação de serviços profissionais, entre outros. Autores como Bellack e Hersen, Matson, Sevin e Box (citado por Del Prette \& Del Prette, 2005) têm expressado preocupação com as consequências desenvolvimentais dos défices de habilidades sociais em etapas formativas da vida do indivíduo, reconhecendo que podem comprometer fases posteriores do seu ciclo vital. Essa preocupação é reforçada pelas evidências de correlação entre os défices e uma variedade de problemas psicológicos, como a delinquência juvenil, o desajustamento escolar, o suicídio, problemas conjugais e consumo de droga(s), além de síndromes clínicas como depressão e a esquizofrenia.

A evidência da sua importância para a qualidade de vida, de saúde, para além de satisfação pessoal e profissional, é mostrada nos estudos realizados por Del Prette e Del Prette (2001), que revelam que as deficiências nas habilidades sociais estão relacionadas com uma qualidade de vida inferior e com diversos tipos de transtornos psicológicos como a timidez, o isolamento social, a delinquência juvenil, o desajustamento escolar, o suicídio e problemas conjugais, bem como as neuroses histéricas, o uso de drogas e o alcoolismo (Hidalgo \& Abarca, 1992).

Relativamente à relação entre o uso de drogas e as habilidades sociais, estudos brasileiros revelaram a existência de relações entre o défice das habilidades sociais e o uso de álcool e drogas. Estes estudos demonstraram que os dependentes de substâncias apresentam índices de habilidades sociais abaixo da média, quando comparados com a média populacional divulgada no inventário das habilidades sociais (Del Prette \& Del Prette, 2001; Correa, 2003; Lopes, 2003; Barkin, Smith \& Durant, 2002; Gaffney, Thorpe, Young, Collet \& Occhipinti, 1998). Face a isto, Del Prette e Del Prette (2005) consideram que o treino das habilidades sociais é indispensável nos tratamentos dos sujeitos com problemas de abuso de álcool e de drogas - além disso, a sua eficácia está a ser estudada não apenas no tratamento, mas também na prevenção dos transtornos por uso de substâncias psicoactivas. O estudo em questão teve assim como objectivo avaliar as condutas antissociais e delitivas em toxicodependentes. Espera-se que os toxicodependentes apresentem índices superiores de condutas antissociais e delitivas em comparação com o grupo não consumidor de substâncias psicoactivas ilícitas.

\section{Método}

\section{Amostra}

Compuseram a amostra 124 sujeitos do sexo masculino e do sexo feminino, divididos em três grupos: 61 sujeitos não consumidores de droga (não dependentes de drogas ilícitas - grupo de controlo (GC)), 32 indivíduos toxicodependentes em tratamento em comunidades terapêuticas (CT) e por 31 sujeitos toxicodependentes em programa de substituição opiácea de baixo limiar (PSOBLE). Em relação a idade, o grupo de não consumidores de drogas apresentou uma média de 27,57 anos (DP = 6,44), seguido do grupo de CT $(\mathrm{M}=34,00 ; \mathrm{DP}=$ $5,88)$ e por fim pelo grupo de PSOBLE $(\mathrm{M}=36,45$; $\mathrm{DP}=7,33)($ Tabela 4). 


\section{Instrumentos}

O questionário foi composto pelos seguintes instrumentos:

Atitudesface à delinquência: avaliada a partir da Attitude Toward Delinquency Scale (ATDS) de Loeber, Farrington, Southamer-Lober e Van Kammen(1998). A ATDS é constituída por 11 itens, sendo a resposta dada por uma escala tipo Likert de 5 pontos, permitindo a cada indivíduo optar entre (1) Concordo muito, (2) Concordo, (3) Não concordo nem discordo, (4) Discordo e (5) Discordo muito. Os resultados variam de 11 a 55 , sendo que os resultados mais elevados indicam maiores níveis de atitudes face à delinquência. No que se refere às qualidades psicométricas da escala, o APDS revela ter uma boa consistência interna, uma vez que o valor $\alpha$ de Cronbach total foi de 0.91 .

Crenças sobre a agressão e alternativas: avaliada a partir da Beliefs about Aggression and Alternatives Scale (BAAS) de Farrel, Meyer e White (2001), pretende avaliar as crenças dos sujeitos acerca do uso da agressão e do apoio de respostas violentas a situações hipotéticas. A escala é composta por 12 itens, com formato de resposta tipo Likert de cinco pontos, (1) Concordo muito, (2) Concordo, (3) Não concordo nem discordo, (4) Discordo e (5) Discordo muito. Os resultados variam de 12 a 60, nos quais os resultados mais elevados indicam maiores níveis de crenças. Relativamente às qualidades psicométricas, a BAAS detém de uma boa consistência interna, com o valor $\alpha$ de Cronbach para crenças sobre a agressão de 0.72 e para o uso de estratégias não violentas de 0.72 .

Condutas antissociais e delitivas ou delinquentes: avaliada por meio da Escala de Condutas Antissociais e Delitivas (ECAD) proposta por Seisdedos (1988), adaptada por Sintra e Lopes (2006), é uma medida comportamental relacionada com condutas antissociais e delitivas, composta por 40 itens, sendo a resposta dada por uma escala tipo Likert de 10 pontos (0 - Nunca; 9 - Sempre). É constituída pela subescala Condutas Antissociais, composta por 20 itens $(1,2,4,5,9,10,11,12,15,18,21,24,26,28,29$, $30,34,35,37,38)$ que não revelam delitos mas comportamentos que desafiam a ordem social e violam as normas sociais (por exemplo: atirar lixo ao chão quando há um cesto do lixo perto; tocar a campainha da casa de alguém e sair a correr), e pela subescala Condutas Delitivas, composta por 20 itens $(3,6,7$, $8,13,14,16,17,19,20,22,23,25,27,31,32,33,36$,
39, 40) que reúnem os comportamentos delitivos que estão contra a lei, exprimindo uma infracção ou uma conduta delitiva ou prejudicial a alguém ou mesmo à sociedade como um todo (por exemplo: roubar objectos dos carros; conseguir dinheiro assustando as pessoas mais fracas). Os resultados variam de $0 \mathrm{a}$ 360 , sendo que os resultados mais elevados são um indicador de maiores níveis de condutas antissociais e delitivas. A Escala das Condutas Antissociais e Delitivas evidenciou valores de consistência para as duas subescalas, relativamente às condutas antissociais foi encontrado um $\alpha$ de Cronbach de 0.86 e de 0.92 para as condutas delitivas ou delinquentes. A análise factorial confirmatória realizada por meio do Lisrel 8.0 corroborou estas dimensões previamente encontradas $\left(\chi^{2} / \mathrm{gl}=1,35\right.$; AGFI $=0,89 ; \mathrm{PHI}(\varphi)=$ $0,79, \mathrm{p}>0,05)$ (Formiga \& Gouveia, 2003).

Habilidades sociais: avaliadas por meio do Inventário de Habilidades Sociais (IHS), elaborado e validado por Del Prette e Del Prette em 2001, é uma medida de autorrelato que avalia o reportório de comportamentos sociais usualmente pedidos em situações interpessoais quotidianas para um desempenho competente, ou seja, é um instrumento que permite identificar défices e recursos em habilidades sociais. O IHS é composto por 42 itens, cada um deles descreve uma relação interpessoal e uma possível reacção diante de uma determinada situação. Os sujeitos são solicitados a indicar com que frequência se comportam da forma descrita em cada um dos itens, de acordo com uma escala tipo Likert de 5 pontos, que varia de (1) nunca a (5) sempre ou quase sempre. Grande parte dos itens éredigida de modo a que a frequência mais elevada na reacção indique défice na habilidade social pertinente para determinada situação. Quanto às qualidades psicométricas, os dados apresentados no manual demonstraram índices adequados de consistência interna, $\alpha$ de Cronbach de 0,75, estabilidade teste-reteste $(r=0,90 ; p<0,01)$ e validade convergente com o Inventário de Assertividade de Rathus ( $\mathrm{r}=$ $0,79, \mathrm{p}<0,01) . \mathrm{Na}$ análise factorial foram obtidos seis factores, embora o sexto não inclua itens com carga factorial relevante, cada um avalia um conjunto de habilidades sociais. O factor 1 (itens 1, 5, 7, 11, 12, 14, 15, 16, 20, 21 e 29), denominado de enfrentamento/ autoafirmação com risco, avalia as habilidades do sujeito se apresentar a outra pessoa, abordar uma relação sexual, discordar da autoridade, discordar do grupo, lembrar a um amigo uma dívida, declarar o seu amor, 
lidar com críticas injustas, fazer uma exposição a uma plateia conhecida, manter uma conversa com desconhecidos e fazer perguntas a desconhecidos. É um factor que se refere ao conceito de assertividade. O factor 2 (itens 3, 6, 8, 10, 28, 30 e 35), designado de autoafirmação na expressão do sentimento positivo, avalia as habilidades de elogiar familiares e outras pessoas, expressar sentimentos positivos, agradecer elogios, defender uma pessoa do grupo e participar numa conversa. Podemos dizer que é um factor que expõe situações que retratam expressões de afecto positivo e afirmações de autoestima e apresentam um risco mínimo de rejeição/punição. O factor 3 (itens 13, 17, 19, 22, 24, 36 e 37), intitulado de conversação e desenvolvimento social, avalia as habilidades de manter e terminar conversas de cara a cara, encerrar conversas ao telefone, abordar as autoridades, reagir a elogios, pedir favores a colegas e recusar pedidos inconvenientes. Este factor reúne, desta forma, itens relacionados com as situações sociais que envolvem comportamentos verbais e supõem o conhecimento das normas básicas que regulam as interacções interpessoais. O factor 4 (itens 9, 23 e 26), denominado de autoexposição a desconbecidos e situaçoes novas, avalia as habilidades de fazer apresentações ou conferências em público e pedir favores ou fazer perguntas a desconhecidos - inclui, assim, itens que envolvem a abordagem a desconhecidos. O factor 5 (itens 18, 31 e 38), designado de autocontrolo da agressividade, avalia as habilidades de lidar com as críticas dos pais, gozos ou brincadeiras ofensivas e cumprimentar pessoas desconhecidas por impulsividade-é, assim, um factor que reúne itens que investigam reacções a estímulos aversivos do interlocutor que disputam o controlo da raiva e da agressividade. Os restantes itens não se encontram em nenhum factor. É de salientar que nos itens 2, 8, 9, 13, 17, 18, 19, 22, 23, 24, 26, 34, 36 e 37 , a pontuação será posteriormente invertida para a obtenção dos escores, e que todos os factores evidenciaram valores de consistência interna - o factor um obteve um $\alpha$ de Cronbach 0,96, o dois 0,86 , o três 0,81 , o quarto 0,75 e o cinco 0,71 .

Atitudes perante as mulheres: avaliada pela Escala de Atitudes Profeministas, versão reduzida da Atittudes Toward Women Scale (AWS; Spence, J., Helmreich, 1978a). A escala é composta por 15 itens, com formato de resposta tipo Likert de 4 pontos, na qual (A) Concordo totalmente, (B) Concordo (C) Discordo e (D) Discordo totalmente, variando a pontuação total entre 15 e 60 , com os valores mais elevados a corresponder a um maior grau de profeminismo e atitudes igualitárias (Spence \& Helmreich, 1978). Trata-se de uma medida de autoavaliação das atitudes perante as mulheres, que contém itens que descrevem os direitos, papéis e privilégios das mulheres, sendo solicitado o seu grau de concordância em relação a estas afirmações. A escala evidenciou valores de consistência interna, $\alpha$ de Cronbach de 0,89 - para além deste facto, foi ainda encontrada uma correlação de 0,91 entre a versão reduzida da AWS (1978) e a versão original desta escala (AWS, Spence \& Helmreich, 1972).

Dados sociodemográficos: recolhidos por meio de um conjunto de questões destinadas a obter informações referentes às variáveis idade, data de nascimento, sexo, naturalidade, nacionalidade, etnia, estado civil, habilitações literárias, situação profissional, número de irmãos, situação conjugal dos pais, coabitação na infância, bem como questões relacionadas com os hábitos de consumo e sexualidade.

\section{Procedimento}

Este estudo transversal e comparativo foi realizado com medidas de autopreenchimento, no qual foi solicitada a colaboração voluntária dos indivíduos para participar na investigação. De igual forma, foram realizadas explicações prévias sobre a natureza e objectivos do estudo. Face à especificidade deste, foram ainda minorados os possíveis receios ou desconfianças dos participantes assegurando-lhes os aspectos éticos relativos à confidencialidade e ao anonimato dos dados. $\mathrm{Na}$ existência de dúvidas, estas foram esclarecidas individualmente. Após explicação sobre o objectivo do estudo e esclarecimento de dúvidas, os questionários foram preenchidos em pequenos grupos pela seguinte ordem: Dados sociodemográficos, ATDS, BAAS, ECAD, IHS e AWS, sendo que a sua administração demorou cerca de 20 minutos. A amostra foi recolhida entre janeiro e março de 2007 nas comunidades terapêuticas "Ares do Pinhal" e "Ponte", bem como no programa de substituição opiácea em baixo limiar-Centro de Acolhimento de Alcântara. O grupo de controlo foi constituído por pessoas que referiram nunca ter sido dependentes de substâncias psicoactivas ilícitas.

Para a análise dos dados o pacote estatístico SPSSWIN, em sua versão 15.0, foi utilizado para 
tabular os dados e realizar as análises estatísticas descritivas, bem como os cálculos referentes à correlação de Pearson e análises de variância (ANOVA).

\section{Resultados}

A fim de atender aos objetivos do presente estudo, bem como para facilitar a compreensão do leitor, optou-se em apresentar os dados por etapa:

Em relação aos dados sociodemográficos (Tabela 1), observou-se diferença significativa entre os grupos nas variáveis seguintes: estado civil $\left(\chi^{2}(10)\right.$ $=28,632 ; \mathrm{p} \leq 0,001)$, o que se traduz pelo facto de a maior parte dos toxicodependentes em CT $(77,4 \%)$ e do GC (70,5\%) serem solteiros, enquanto $15,6 \%$ dos toxicodependentes em PSOBLE referirem estar separados; na variável babilitações literárias $\left(\chi^{2}(12)=\right.$ $71,265 ; \mathrm{p} \leq 0,001)$, uma vez que se verificou que a maior parte dos sujeitos do GC $(57,4 \%)$ apresenta o secundário completo ( $12^{\circ}$ ano) enquanto que os toxicodependentes, quer em CT $(41,9 \%)$, quer em PSOBLE (37,5\%), apresentaram maioritariamente o $3^{\circ}$ ciclo ( $9^{\circ}$ ano); na variável situação profissional $\left(\chi^{2}(8)=\right.$ 57,932; $\mathrm{p} \leq 0,001)$, no sentido de que a maior parte dos toxicodependentes em CT $(51,6 \%)$ e em PSOBLE $(40,6 \%)$ estava desempregada em comparação com o GC $(62,7 \%)$, cujos membros apresentavam um emprego estável; na variável situação conjugal dospais $\left(\chi^{2}\right.$ (8) $=37,070 ; p \leq 0,001)$, o que se traduz pelo facto de a maior parte dos pais do GC $(75,4 \%)$ e dos toxicodependentes em CT (32,3\%) estarem casados/em união de facto, enquanto que naqueles em PSOBLE existe uma percentagem maior de pais separados/ divorciados (28,1\%); na variável relação actual com a família $\left(\chi^{2}(6)=49,792 ; \mathrm{p} \leq 0,001\right)$, o que se explica pelo facto de a grande maioria do GC (88,5\%) referir uma boa relação com a família, enquanto que nos toxicodependentes em PSOBLE $(56,7 \%)$ e em CT $(48,4 \%)$ também se evidenciou maioritariamente uma relação satisfatória com a família, porém não tão significativa como a evidenciada pelo GC; na variável tem familiares dependentes $\left(\chi^{2}(2)=15,198\right.$; $\mathrm{p} \leq 0,001)$, no sentido de que $100 \%$ do GC não refere qualquer familiar dependente de drogas, comparativamente com o grupo de toxicodependentes em CT $(96,7 \%)$ e em PSOBLE (80,0\%). A maioria dos grupos da amostra são de meio urbano, portugueses, caucasianos, não têm pais emigrantes e coabitaram na infância com os pais (ver, novamente, Tabela 1).
Quanto aos comportamentos de risco, foram encontradas diferenças estatisticamente significativas entre os toxicodependentes para a variável é seropositivo $\left(\chi^{2}(2)=104,279 ; \mathrm{p} \leq 0,001\right)$, uma vez que a percentagem dos sujeitos não seropositivos foi mais elevada nos toxicodependentes em CT $(64,5 \%)$ do que naqueles em PSOBLE (50,0\%) (Tabela 2).

Relativamente à situação judicial, encontraram-se diferenças estatisticamente significativas para as variáveis: já esteve detido $\left(\chi^{2}(2)=50,638 ; \mathrm{p} \leq 0,001\right)$, o que se traduz pelo facto de os toxicodependentes em CT terem apresentado uma percentagem mais elevada $(63,3 \%)$ quando comparados com aqueles em PSOBLE (59,4\%): já teve julgamentos $\left(\chi^{2}(2)=69,405\right.$; $\mathrm{p} \leq 0,001)$, com os toxicodependentes em CT a apresentarem uma percentagem mais elevada de julgamentos $(80,6 \%)$ em comparação com os toxicodependentes em PSOBLE (73,3\%); já tevepena suspensa $\left(\chi^{2}(2)=46,62\right.$; $p \leq 0,001)$, no sentido de que os toxicodependentes em CT apresentam uma percentagem mais elevada de pena suspensa $(63,3 \%)$ quando comparados com aqueles em PSOBLE (39,3\%); bem como para a variável prisão efectiva $\left(\chi^{2}(2)=31,227 ; \mathrm{p} \leq 0,001\right)$, o que se traduz pelo facto de os toxicodependentes em CT terem se revelado o grupo que apresentou uma maior percentagem $(42,5 \%)$ em comparação com o grupo em PSOBLE (33,3\%) (Tabela 3).

Observou-se diferença significativa entre os grupos para a variável idade $(H=36,619$; $\leq \leq 0,001)$, no sentido de os sujeitos do GC terem apresentado valores médios inferiores de idade $(\mathrm{M}=27.57$; $\mathrm{DP}=6,44)$ comparativamente com os indivíduos toxicodependentes em PSOBLE $(\mathrm{M}=34,00 ; \mathrm{DP}=$ $5,88)$ e em CT $(M=36,45 ; \mathrm{DP}=7,33)$. No que se refere à variável com que idade deixou a escola, também observamos diferenças estatisticamente significativas entre os grupos $(H=52,085 ; p \leq 0,001)$, com o GC a deixar a escola em média mais tarde $(\mathrm{M}=23,15$; DP $=5,12)$ em comparação com o grupo em PSOBLE $(\mathrm{M}=16,77 ; \mathrm{DP}=3,69)$ e com o grupo em CT $(\mathrm{M}$ $=15,93 ; \mathrm{DP}=3,33)($ Tabela 4$)$.

Quanto aos consumos de substâncias ilícitas, encontraram-se diferenças estatisticamente significativas entre os dois grupos de toxicodependentes para a variável total de anos de consumo $(t=0,004 ; \mathrm{p} \leq$ $0,001)$, o que se traduz pelo facto de os toxicodependentes em CT apresentarem uma média total de anos de consumo $(M=18,19$; $D P=7,27)$ superior à dos toxicodependentes em PSOBLE $(\mathrm{M}=13,31$; $\mathrm{DP}=6,66)$, e para a variável total de anos de consumo 
Sintra, C. I. F., Lopes, P., \& Formiga, N.

Tabela 1 - Variáveis sociodemográficas

(Continua)

\begin{tabular}{|c|c|c|c|c|c|c|c|}
\hline \multirow{2}{*}{ Grupos } & \multicolumn{2}{|c|}{ Controlo } & \multicolumn{2}{|c|}{$\begin{array}{l}\text { Toxicodependentes em } \\
\text { redução de risco }\end{array}$} & \multicolumn{2}{|c|}{$\begin{array}{l}\text { Toxicodependentes em } \\
\text { comunidade terapêutica }\end{array}$} & \multirow[b]{2}{*}{$\chi^{2}$} \\
\hline & $\mathrm{n}$ & $\%$ & $\mathrm{n}$ & $\%$ & $\mathrm{n}$ & $\%$ & \\
\hline Sexo & & & & & & & $17,065^{* * *}$ \\
\hline Masculino & 28 & 45,9 & 18 & 56,3 & 28 & 90,3 & \\
\hline Feminino & 33 & 54,1 & 14 & 43,8 & 3 & 9,7 & \\
\hline Naturalidade & & & & & & & 5,904 \\
\hline Meio urbano & 37 & 62,7 & 26 & 83,9 & 25 & 80,6 & \\
\hline Meio rural & 22 & 37,3 & 5 & 16,1 & 6 & 19,4 & \\
\hline Nacionalidade & & & & & & & 4,079 \\
\hline Portuguesa & 57 & 93,4 & 28 & 87,5 & 31 & 100,0 & \\
\hline Outra & 4 & 6,6 & 4 & 12,5 & 0 & 0,0 & \\
\hline Etnia & & & & & & & 5,107 \\
\hline Caucasiana & 55 & 94,8 & 23 & 79,3 & 28 & 90,3 & \\
\hline Negroide & 3 & 5,2 & 6 & 20,7 & 3 & 9,7 & \\
\hline Estado civil & & & & & & & $28,632^{* * *}$ \\
\hline Solteiro & 43 & 70,5 & 22 & 68,8 & 24 & 77,4 & \\
\hline União de facto & 3 & 4,9 & 0 & 0,0 & 0 & 0,0 & \\
\hline Casado & 13 & 21,3 & 0 & 0,0 & 1 & 3,2 & \\
\hline Separado & 0 & 0,0 & 5 & 15,6 & 4 & 12,9 & \\
\hline Divorciado & 2 & 3,3 & 4 & 12,5 & 2 & 6,5 & \\
\hline Viúvo & 0 & 0,0 & 1 & 3,1 & 0 & 0,0 & \\
\hline Habilitações literárias & & & & & & & $71,265^{* * *}$ \\
\hline $\begin{array}{l}\text { Sem escolaridade, } \\
\text { mas sabe ler e } \\
\text { escrever }\end{array}$ & 0 & 0,0 & 1 & 3,1 & 0 & 0,0 & \\
\hline $\begin{array}{l}1^{\circ} \text { Ciclo (escola } \\
\text { primária) }\end{array}$ & 0 & 0,0 & 3 & 9,4 & 5 & 16,1 & \\
\hline $\begin{array}{l}2^{\circ} \text { Ciclo (ciclo } \\
\text { preparatório) }\end{array}$ & 3 & 4,9 & 10 & 31,3 & 9 & 29,0 & \\
\hline $3^{\circ}$ Ciclo ( $9^{\circ}$ ano $)$ & 4 & 6,6 & 12 & 37,5 & 13 & 41,9 & \\
\hline Secundário $\left(12^{\circ}\right.$ ano $)$ & 35 & 57,4 & 5 & 15,6 & 4 & 12,9 & \\
\hline $\begin{array}{l}\text { Bacharelato/ } \\
\text { Licenciatura }\end{array}$ & 13 & 21,3 & 0 & 0,0 & 0 & 0,0 & \\
\hline Curso profissional & 6 & 9,8 & 1 & 3,1 & 0 & 0,0 & \\
\hline
\end{tabular}


Tabela 1 - Variáveis sociodemográficas

(Conclusão)

\begin{tabular}{|c|c|c|c|c|c|c|c|}
\hline \multirow{2}{*}{ Grupos } & \multicolumn{2}{|c|}{ Controlo } & \multicolumn{2}{|c|}{$\begin{array}{l}\text { Toxicodependentes em } \\
\text { redução de risco }\end{array}$} & \multicolumn{2}{|c|}{$\begin{array}{l}\text { Toxicodependentes em } \\
\text { comunidade terapêutica }\end{array}$} & \multirow[b]{2}{*}{$\chi^{2}$} \\
\hline & $\mathrm{n}$ & $\%$ & $\mathrm{n}$ & $\%$ & $\mathrm{n}$ & $\%$ & \\
\hline Situação profissional & & & & & & & $57,932^{* * *}$ \\
\hline S/profissão & 13 & 22,0 & 15 & 46,9 & 6 & 19,4 & \\
\hline Desempregado & 1 & 1,7 & 13 & 40,6 & 16 & 51,6 & \\
\hline Emprego ocasional & 8 & 13,6 & 2 & 6,3 & 2 & 6,5 & \\
\hline Emprego estável & 37 & 62,7 & 1 & 3,1 & 6 & 19,4 & \\
\hline $\begin{array}{l}\text { Curso de formação } \\
\text { subsidiado }\end{array}$ & 0 & 0,0 & 1 & 3,1 & 1 & 3,2 & \\
\hline $\begin{array}{l}\text { Situação conjugal dos } \\
\text { pais }\end{array}$ & & & & & & & $37,070 * * *$ \\
\hline $\begin{array}{l}\text { Casados/união de } \\
\text { facto }\end{array}$ & 46 & 75,4 & 8 & 25,0 & 10 & 32,3 & \\
\hline $\begin{array}{l}\text { Divorciados/ } \\
\text { separados }\end{array}$ & 8 & 13,1 & 9 & 28,1 & 4 & 12,9 & \\
\hline Mãe falecida & 0 & 0,0 & 6 & 18,8 & 7 & 22,6 & \\
\hline Pai falecido & 6 & 9,8 & 4 & 12,5 & 6 & 19,4 & \\
\hline Ambos falecidos & 1 & 1,6 & 5 & 15,6 & 4 & 12,9 & \\
\hline Pais emigrantes & & & & & & & 0,835 \\
\hline Não & 51 & 83,6 & 23 & 76,7 & 24 & 77,4 & \\
\hline $\operatorname{Sim}$ & 10 & 16,4 & 7 & 23,3 & 7 & 22,6 & \\
\hline $\begin{array}{l}\text { Coabitação na } \\
\text { infância }\end{array}$ & & & & & & & 0,974 \\
\hline Pais & 58 & 98,3 & 27 & 100,0 & 28 & 96,6 & \\
\hline Outros familiares & 1 & 1,7 & 0 & 0,0 & 1 & 3,4 & \\
\hline $\begin{array}{l}\text { Relação actual com a } \\
\text { família }\end{array}$ & & & & & & & $49,792 * * *$ \\
\hline Boa & 54 & 88,5 & 7 & 23,3 & 9 & 29,0 & \\
\hline Má & 1 & 1,6 & 2 & 6,7 & 3 & 9,7 & \\
\hline Satisfatória & 6 & 9,8 & 17 & 56,7 & 15 & 48,4 & \\
\hline Sem relação & 0 & 0,0 & 4 & 13,3 & 4 & 12,9 & \\
\hline $\begin{array}{l}\text { Tem familiares } \\
\text { dependentes }\end{array}$ & & & & & & & $15,198^{* * *}$ \\
\hline Não & 61 & 100,0 & 24 & 80,0 & 29 & 96,7 & \\
\hline Sim & 0 & 0,0 & 6 & 20,0 & 1 & 3,3 & \\
\hline
\end{tabular}

Legenda: $* * * \mathrm{p} \leq 0,001$. 
Tabela 2 - Comportamentos de risco por grupos de toxicodependentes

\begin{tabular}{|c|c|c|c|c|c|}
\hline \multirow{2}{*}{ Grupos } & \multicolumn{2}{|c|}{$\begin{array}{l}\text { Toxicodependentes em } \\
\text { redução de risco }\end{array}$} & \multicolumn{2}{|c|}{$\begin{array}{l}\text { Toxicodependentes em } \\
\text { comunidade terapêutica }\end{array}$} & \multirow[b]{2}{*}{$\chi^{2}$} \\
\hline & $\mathbf{n}$ & $\%$ & $\mathrm{n}$ & $\%$ & \\
\hline Usa preservativo nas relações sexuais & & & & & 3,855 \\
\hline Sempre & 17 & 53,1 & 10 & 32,3 & \\
\hline Às vezes & 13 & 40,6 & 20 & 64,5 & \\
\hline Nunca & 2 & 6,3 & 1 & 3,2 & \\
\hline Já partilhou material de consumo & & & & & 9,404 \\
\hline Seringa & 4 & 16,0 & 0 & 0,0 & \\
\hline Algodão & 3 & 12,0 & 2 & 7,4 & \\
\hline Recipiente/colher & 3 & 12,0 & 1 & 3,7 & \\
\hline Tubo de inalar & 2 & 8,0 & 4 & 14,8 & \\
\hline Todas & 6 & 24,0 & 9 & 33,3 & \\
\hline Seringa, algodão e recipiente/colher & 2 & 8,0 & 4 & 14,8 & \\
\hline Algodão, recipiente/colher e tubo de inalar & 2 & 8,0 & 2 & 7,4 & \\
\hline Algodão e recipiente/colher & 1 & 4,0 & 3 & 11,1 & \\
\hline Algodão e tubo de inalar & 1 & 4,0 & 0 & 0,0 & \\
\hline Tubo de inalar e recipiente/colher & 1 & 4,0 & 2 & 7,4 & \\
\hline É seropositivo & & & & & $28,022^{* * *}$ \\
\hline Sim & 16 & 50,0 & 11 & 35,5 & \\
\hline Não & 16 & 50,0 & 20 & 64,5 & \\
\hline
\end{tabular}

Legenda: $* * * \mathrm{p} \leq 0,001$.

Tabela 3 - Situação judicial por grupos de toxicodependentes

(Continua)

\begin{tabular}{|c|c|c|c|c|c|}
\hline \multirow[t]{2}{*}{ Grupos } & \multicolumn{2}{|c|}{$\begin{array}{l}\text { Toxicodependentes em } \\
\text { redução de risco }\end{array}$} & \multicolumn{2}{|c|}{$\begin{array}{l}\text { Toxicodependentes em } \\
\text { comunidade terapêutica }\end{array}$} & \multirow[b]{2}{*}{$\chi^{2}$} \\
\hline & $\mathrm{n}$ & $\%$ & $\mathrm{n}$ & $\%$ & \\
\hline Já esteve detido & & & & & $50,638^{* * *}$ \\
\hline Não & 13 & 40,6 & 11 & 36,7 & \\
\hline $\operatorname{Sim}$ & 19 & 59,4 & 19 & 63,3 & \\
\hline Já teve julgamentos & & & & & $69,405^{* * *}$ \\
\hline Não & 8 & 26,7 & 6 & 19,4 & \\
\hline $\operatorname{Sim}$ & 22 & 73,3 & 25 & 80,6 & \\
\hline Já teve pena suspensa & & & & & $46,629 * * *$ \\
\hline Não & 17 & 60,7 & 11 & 36,7 & \\
\hline
\end{tabular}


Tabela 3 - Situação judicial por grupos de toxicodependentes

(Conclusão)

\begin{tabular}{|c|c|c|c|c|c|}
\hline \multirow{2}{*}{ Grupos } & \multicolumn{2}{|c|}{$\begin{array}{l}\text { Toxicodependentes em } \\
\text { redução de risco }\end{array}$} & \multicolumn{2}{|c|}{$\begin{array}{l}\text { Toxicodependentes em } \\
\text { comunidade terapêutica }\end{array}$} & \multirow[b]{2}{*}{$\chi^{2}$} \\
\hline & $\mathbf{n}$ & $\%$ & $\mathbf{n}$ & $\%$ & \\
\hline Sim & 11 & 39,3 & 19 & 63,3 & \\
\hline Prisão efectiva & & & & & $31,227 * * *$ \\
\hline Não & 20 & 66,7 & 17 & 54,8 & \\
\hline $\operatorname{Sim}$ & 10 & 33,3 & 14 & 45,2 & \\
\hline
\end{tabular}

Legenda: $* * * \mathrm{p} \leq 0,001$

Tabela 4 - Diferenças entre grupos face à variável idade, idade com que deixou de andar na escola, idade que tinha quando a mãe faleceu e idade que tinha quando o pai faleceu

\begin{tabular}{|c|c|c|c|c|c|c|c|c|c|c|}
\hline \multirow{2}{*}{ Grupos } & \multicolumn{3}{|c|}{ Controlo } & \multicolumn{3}{|c|}{$\begin{array}{l}\text { Toxicodependentes em } \\
\text { redução de risco }\end{array}$} & \multicolumn{3}{|c|}{$\begin{array}{l}\text { Toxicodependentes em } \\
\text { comunidade terapêutica }\end{array}$} & \multirow[b]{2}{*}{$\mathrm{p}$} \\
\hline & $\mathrm{n}$ & $\mathbf{M}$ & DP & $\mathrm{n}$ & $\mathbf{M}$ & DP & $\mathbf{n}$ & $\mathbf{M}$ & DP & \\
\hline Idade do sujeito & 61 & 27,57 & 6,44 & 32 & 34,00 & 5,88 & 31 & 36,45 & 7,33 & ,000 \\
\hline $\begin{array}{l}\text { Com que idade } \\
\text { deixou a escola }\end{array}$ & 61 & 23,15 & 5,12 & 31 & 16,77 & 3,69 & 30 & 15,93 & 3,33 & ,000 \\
\hline $\begin{array}{l}\text { Que idade tinha } \\
\text { quando a mãe faleceu }\end{array}$ & 1 & 51,00 & - & 10 & 20,10 & 10,92 & 11 & 22,45 & 13,46 & ,082 \\
\hline $\begin{array}{l}\text { Que idade tinha } \\
\text { quando o pai faleceu }\end{array}$ & 6 & 25,50 & 12,33 & 8 & 20,75 & 9,68 & 7 & 26,29 & 12.35 & ,603 \\
\hline
\end{tabular}

por via endovenosa / injectada $(t=6,292 ; p \leq 0,05)$, o que se explica pelo facto de os toxicodependentes em CT apresentarem uma média total de anos de consumo por via endovenosa/injectada de 13,56 anos (DP = $8,63)$, bastante superior à dos toxicodependentes em PSOBLE ( $\mathrm{M}=8,38 ; \mathrm{DP}=5,69)$. Embora sem diferenças estatisticamente significativas, torna-se pertinente salientar que os toxicodependentes em PSOBLE iniciam mais cedo o consumo de heroína $(\mathrm{M}=19,19 ; \mathrm{DP}=5,07)$, de cocaína $(\mathrm{M}=19,69$; $\mathrm{DP}=5,52)$ e por via endovenosa/injectada $(\mathrm{M}=$ 21,50; DP $=7,96)$, quando comparados com os toxicodependentes em CT $(\mathrm{M}=18,90 ; \mathrm{DP}=4,05)$, $(\mathrm{M}=19,45 ; \mathrm{DP}=4,40)$ e $(\mathrm{M}=20,12 ; \mathrm{DP}=8,63)$ (Tabela 5).
Por outro lado, os toxicodependentes em CT apresentam uma percentagem superior de tratamentos anteriores $(87,1 \%)$ comparativamente com os toxicodependentes em PSOBLE (71,9\%) (Tabela 6).

\section{Análise das diferenças entre grupos para a AWS}

Foram encontradas diferenças estatisticamente significativas na AWS $[\mathrm{F}(1,121)=4,08 ; \mathrm{p} \leq$ $0,01]$ em função dos grupos, tendo-se verificado que o grupo dos toxicodependentes em PSOBLE $(\mathrm{M}=$ 2,38; DP $=0,28)$ apresenta resultados significativamente mais baixos (menos tolerantes) que o grupo 
de toxicodependentes em CT $(\mathrm{M}=2,44 ; \mathrm{DP}=0,23)$ e o GC $(M=2,52$; DP $=0,20)$. Por meio do Post Hoc-Tukey verificou-se que estas diferenças foram significativas entre o GC e os toxicodependentes em PSOBLE (ver Tabela 7).

\section{Análise das diferenças entre grupos para a ECAD}

Quanto às condutas antissociais e delitivas, existem diferenças estatisticamente significativas, quer nas condutas antissociais $[\mathrm{F}(2,119)=6,28$; $\mathrm{p} \leq 0,01]$, quer nas condutas delitivas $[\mathrm{F}(2,120)=$ $16,84 ; \mathrm{p} \leq 0,001]$, quer nas condutas antissociais e delitivas $[\mathrm{F}(2,121)=13,57 ; \mathrm{p} \leq 0,001]$, com o GC a apresentar valores médios inferiores, de 24,38 (DP $=18,45)$ para as condutas antissociais, de 4,98 (DP $=8,74)$ para as condutas delitivas e de $0,75(\mathrm{DP}=$ $0,65)$ para as condutas antissociais e delitivas, quando comparados com os grupos de toxicodependentes em CT $(\mathrm{M}=37,00 ; \mathrm{DP}=21,93 ; \mathrm{M}=29,30 ; \mathrm{DP}=$ $30,42$ e $\mathrm{M}=1,67$; $\mathrm{DP}=1,23)$ e em PSOBLE $(\mathrm{M}=$ 39,94; $\mathrm{DP}=28,59 ; \mathrm{M}=26,31 ; \mathrm{DP}=28,91$ e $\mathrm{M}=$ $1,76 ; \mathrm{DP}=1,38)($ ver Tabela 8$)$.

\section{Análise das diferenças entre a IHS}

No que respeita à IHS, existem diferenças estatisticamente significativas, quer no enfrentamento/autoafirmação com risco $[\mathrm{F}(2,120)=3,68$; $\mathrm{p} \leq 0,01]$, quer na conversação e desenvolvimento social $[\mathrm{F}(2,119)=4,16 ; \mathrm{p} \leq 0,01]$. No enfrentamento/ autoafirmação com risco, o grupo dos toxicodependentes em CT obteve um valor mais baixo $(\mathrm{M}$ $=32,27 ; \mathrm{DP}=6,41)$, seguido do $\mathrm{GC}(\mathrm{M}=34,62$; $\mathrm{DP}=5,26)$ e do grupo de toxicodependentes em PSOBLE $(\mathrm{M}=36,37$; $\mathrm{DP}=6,81)$. Por meio do teste Post Hoc-Tukey observamos que essas diferenças existem entre o grupo de toxicodependentes em CT e o grupo de toxicodependentes em PSOBLE. No que se refere à conversação e ao desenvolvimento social, o grupo de toxicodependentes em PSOBLE apresentou um valor médio de 21,50 (DP $=5,74$ ), inferior e estatisticamente significativo quando comparado com o $\mathrm{GC}(\mathrm{M}=24,07 ; \mathrm{DP}=3,82)$ e com o grupo de toxicodependentes em CT ( $\mathrm{M}=24,40$; $\mathrm{DP}=4,48)($ ver Tabela 9).

\section{Discussáo}

Foi objectivo do estudo avaliar a relação entre as condutas antissociais e delitivas, agressão e habilidades sociais entre toxicodependentes que se encontram em diferentes dispositivos de ajuda, nomeadamente em programas de redução de riscos e em programas de tratamento, utilizando como grupo de controlo pessoas que nunca foram dependentes de substâncias psicoactivas ilícitas.

Relativamente às condutas antissociais e condutas delitivas, avaliadas pela ECAD, confirmaram-se as hipóteses inicialmente formuladas, ou seja, verificaram-se diferenças estatisticamente significativas entre os toxicodependentes, quer em PSOBLE, quer em CT, que apresentaram níveis médios superiores quando comparados com o GC. Estes resultados vão de encontro com a investigação de Negreiros (1998), que demonstrou a existência de uma relação forte entre o uso de drogas e os comportamentos antissociais. Este resultado pode ainda ser sustentado por meio das afirmações de Brill, citado por Dias (1982), que confirmou nos seus estudos a relação entre a delinquência, a toxicodependência e a história criminal. Tal como referiu Brochu (citado em Morel, Hervé \& Fontaine, 1998), as drogas ilícitas são caras e por isso é preciso muito dinheiro para as consumir regularmente. Isto coloca muitas vezes os toxicodependentes na obrigação de se tornarem delinquentes, o que foi confirmado no estudo de Brochu, pelos valores mais elevados de condutas desviantes (delitivas e antissociais) nos toxicodependentes quando comparados com o grupo de controlo.

Em relação à agressão e alternativas, avaliadas pela BAAS, não se encontraram diferenças estatisticamente significativas entre os toxicodependentes e o grupo de controlo, embora se tenha verificado que os toxicodependentes em PSOBLE apresentaram crenças sobre a agressão e alternativas superiores aos outros dois grupos. Segundo Bushman (citado em Allen et al., 1997), as várias drogas psicoactivas provocam o aumento da agressividade e fazem com que os sujeitos que usam drogas tenham uma probabilidade maior de enveredar por comportamentos agressivos do que aqueles que não usam drogas (Muntaner et al., 1990).

Relativamente às habilidades sociais, avaliadas respectivamente pelo IHS, os nossos resultados 
Tabela 5 - Diferenças entre o grupo de toxicodependentes em redução de risco e o grupo em comunidade terapêutica face à idade de consumo de heroína, idade de consumo de cocaína, total de anos de consumo, início de consumo por via endovenosa/injectada e total de anos de consumo por via endovenosa/injectada

\begin{tabular}{lcccccccc}
\hline \multirow{2}{*}{ Grupos } & \multicolumn{3}{c}{$\begin{array}{c}\text { Toxicodependentes em } \\
\text { redução de risco }\end{array}$} & \multicolumn{4}{c}{$\begin{array}{c}\text { Toxicodependentes em } \\
\text { comunidade terapêutica }\end{array}$} \\
\cline { 2 - 9 } & & $\mathbf{n}$ & $\mathbf{M}$ & $\mathbf{D P}$ & $\mathbf{n}$ & $\mathbf{M}$ & $\mathbf{D P}$ & $\mathbf{p}$ \\
\hline Idade de consumo de heroína & 32 & 19,19 & 5,07 & 30 & 18,90 & 4,05 & 0,807 \\
Idade de consumo de cocaína & 32 & 19,69 & 5,52 & 31 & 19,45 & 4,40 & 0,852 \\
Total de anos de consumo & 32 & 13,31 & 6,66 & 31 & 18,19 & 7,27 & 0,007 \\
Início de consumo por via endovenosa/injectada & 22 & 21,50 & 7,96 & 26 & 20,12 & 5,92 & 0,493 \\
$\begin{array}{l}\text { Total de anos de consumo por via endovenosa/ } \\
\text { injectada }\end{array}$ & 21 & 8,38 & 5,69 & 25 & 13,56 & 8,63 & 0,023 \\
\hline
\end{tabular}

Tabela 6 - Diferenças entre os grupos face aos tratamentos anteriores

\begin{tabular}{lccccc}
\hline & \multicolumn{1}{c}{ Grupos } & \multicolumn{2}{c}{$\begin{array}{c}\text { Toxicodependentes em } \\
\text { redução de risco }\end{array}$} & \multicolumn{2}{c}{$\begin{array}{c}\text { Toxicodependentes em } \\
\text { comunidade terapêutica }\end{array}$} \\
\cline { 2 - 6 } & \multicolumn{1}{c}{$\mathbf{n}$} & $\%$ & $\mathbf{n}$ & $\%$ & $\boldsymbol{\chi}^{\mathbf{2}}$ \\
\hline Já fez algum tratamento anterior? & & & & & 2,228 \\
Não & 9 & 28,1 & 4 & 12,9 \\
Sim & 23 & 71,9 & 27 & 87,1 \\
\hline
\end{tabular}

Tabela 7 - Valores das atitudes perante as mulheres por grupo

\begin{tabular}{lccccc}
\hline & $\mathbf{n}$ & $\mathbf{M}$ & $\mathbf{D P}$ & $\mathbf{F}$ & $\mathbf{p}$ \\
\hline Atitudes perante as mulheres & & & & 4,076 & 0,019 \\
Toxicodependentes PSOBLE $^{\mathrm{a}}$ & 32 & 2,38 & 0,28 & & \\
Toxicodependentes CT $^{\mathrm{a}, \mathrm{b}}$ & 31 & 2,44 & 0,23 & \\
Controlo $^{\text {a }}$ & 61 & 2,52 & 0,20 & \\
\hline
\end{tabular}

Nota: Controlo = grupo de controlo; Toxicodependentes PSOBLE $=$ grupo dos toxicodependentes em programa de substituição opiácea em baixo limiar de exigência; Toxicodependentes CT = grupo dos toxicodependentes em comunidade terapêutica.

Nota: Os índices referem-se aos grupos significativamente diferentes ${ }^{(1)}$. 
apontam para diferenças estatisticamente significativas entre dois dos cinco factores das habilidades sociais considerados pelo IHS, nomeadamente enfrentamento/autoafirmação e conversação e desenvolvimento social, com o grupo de toxicodependentes em CT a apresentar níveis médios inferiores de enfrentamento/autoafirmação e com o grupo de toxicodependentes em PSOBLE a apresentar níveis médios inferiores de conversação e desenvolvimento social.

Tal como refere Miguel (1997, p. 27), sendo esta problemática complexa, não existe uma fórmula universal que permita conduzir à cura desta doença, uma vez que os toxicodependentes são muito diferentes uns dos outros, sendo dissemelhantes na idade, na personalidade, no meio social de origem, no tipo de família, nas substâncias que utilizam, na duração da dependência e no grau de controlo. Face a isto, é fundamental criar uma estratégia adequada à realidade de cada indivíduo, e continuar a estudar a sua especificidade, pois só assim se poderão obter resultados positivos e quem sabe chegar a uma solução para esta problemática.

Este estudo não contemplou os possíveis casos de desordens da personalidade presentes na nossa amostra. Apesar deste estudo e da literatura apontarem na direcção de que existem relações entre o abuso de substâncias ilícitas e as habilidades sociais, considera-se que pesquisas futuras devem continuar a investigar se o défice em habilidades sociais será característico da população de toxicodependentes.

Para concluir, gostávamos de levantar algumas questões: será que os défices nas habilidades sociais funcionam como factores de risco para o consumo de droga(s)? Será que o treino das habilidades sociais pode ser uma estratégia usada no combate ao consumo de droga(s)? Poderá ser uma solução?

Tabela 8 - Valores das condutas antissociais e delitivas por grupo

\begin{tabular}{|c|c|c|c|c|c|}
\hline & $\mathrm{n}$ & $\mathbf{M}$ & DP & F & $\mathrm{p}$ \\
\hline Condutas antissociais & & & & 6,279 & 0,003 \\
\hline Controlo $^{\text {a }}$ & 61 & 24,38 & 18,45 & & \\
\hline Toxicodependentes $\mathrm{CT}^{\mathrm{b}}$ & 29 & 37,00 & 21,93 & & \\
\hline Toxicodependentes PSOBLE ${ }^{\mathrm{b}}$ & 32 & 39,94 & 28,59 & & \\
\hline Condutas delitivas & & & & 16,837 & 0,000 \\
\hline Controlo $^{\text {a }}$ & 61 & 4,98 & 8,74 & & \\
\hline Toxicodependentes PSOBLE ${ }^{\mathrm{b}}$ & 32 & 26,31 & 28,91 & & \\
\hline Toxicodependentes $\mathrm{CT}^{\mathrm{b}}$ & 30 & 29,30 & 30,42 & & \\
\hline Condutas antissociais e delitivas (Nota total) & & & & 13,566 & 0,000 \\
\hline Controlo $^{\text {a }}$ & 61 & 0,75 & 0,65 & & \\
\hline Toxicodependentes $\mathrm{CT}^{\mathrm{b}}$ & 31 & 1,67 & 1,23 & & \\
\hline Toxicodependentes PSOBLE ${ }^{\mathrm{b}}$ & 32 & 1,76 & 1,38 & & \\
\hline
\end{tabular}

Nota: Controlo = grupo de controlo; Toxicodependentes PSOBLE $=$ grupo dos toxicodependentes em programa de substituição opiácea em baixo limiar de exigência; Toxicodependentes CT = grupo dos toxicodependentes em comunidade terapêutica.

Nota: Os índices referem-se aos grupos significativamente diferentes ${ }^{(1)}$. 
Tabela 9 - Valores das habilidades sociais por grupo

\begin{tabular}{|c|c|c|c|c|c|}
\hline & $\mathrm{n}$ & $\mathbf{M}$ & DP & F & $\mathrm{p}$ \\
\hline Enfrentamento/autoafirmação com risco & & & & 3,679 & 0,028 \\
\hline Toxicodependentes $\mathrm{CT}^{\mathrm{a}}$ & 30 & 32,27 & 6,41 & & \\
\hline Controlo $^{\mathrm{a}, \mathrm{b}}$ & 61 & 34,62 & 5,26 & & \\
\hline Toxicodependentes PSOBLE ${ }^{\mathrm{b}}$ & 32 & 36,37 & 6,81 & & \\
\hline Autoafirmação na expressão do sentimento positivo & & & & 0,805 & 0,449 \\
\hline Toxicodependentes $\mathrm{CT}^{\mathrm{a}}$ & 30 & 25,77 & 4,04 & & \\
\hline Toxicodependentes PSOBLE ${ }^{\text {a }}$ & 32 & 26,72 & 3,95 & & \\
\hline Controlo $^{a}$ & 61 & 26,75 & 3,29 & & \\
\hline Conversação e desenvolvimento social & & & & 4,146 & 0,018 \\
\hline Toxicodependentes PSOBLE ${ }^{a}$ & 32 & 21,50 & 5,74 & & \\
\hline Controlo ${ }^{\mathrm{b}}$ & 60 & 24,07 & 3,82 & & \\
\hline Toxicodependentes $\mathrm{CT}^{\mathrm{b}}$ & 30 & 24,40 & 4,48 & & \\
\hline Autoexposição a desconhecidos e situações novas & & & & 0,979 & 0,379 \\
\hline Controlo $^{\text {a }}$ & 61 & 9,48 & 2,44 & & \\
\hline Toxicodependentes $\mathrm{CT}^{\mathrm{a}}$ & 31 & 9,87 & 3,02 & & \\
\hline Toxicodependentes PSOBLE ${ }^{a}$ & 32 & 10,31 & 3,08 & & \\
\hline Autocontrolo da agressividade & & & & 0,171 & 0,843 \\
\hline Toxicodependentes PSOBLE ${ }^{a}$ & 32 & 10,28 & 2,92 & & \\
\hline Controlo $^{a}$ & 61 & 10,54 & 1,92 & & \\
\hline Toxicodependentes $\mathrm{CT}^{\mathrm{a}}$ & 31 & 10,61 & 2,75 & & \\
\hline Habilidades sociais (Nota total) & & & & 1,018 & 0,365 \\
\hline Toxicodependentes $\mathrm{CT}^{\mathrm{a}}$ & 31 & 3,20 & 0,39 & & \\
\hline Controlo $^{\text {a }}$ & 61 & 3,28 & 0,28 & & \\
\hline Toxicodependentes PSOBLE ${ }^{a}$ & 32 & 3,31 & 0,28 & & \\
\hline
\end{tabular}

Nota: Controlo = grupo de controlo; Toxicodependentes PSOBLE = grupo dos toxicodependentes em programa de substituição opiácea em baixo limiar de exigência; Toxicodependentes CT = grupo dos toxicodependentes em comunidade terapêutica.

Nota: Os índices referem-se aos grupos significativamente diferentes ${ }^{(1)}$. 


\section{Referências}

Abraão, I. (1999). Factores de risco e factores protectores para as toxicodependências. Uma breve revisão. Toxicodependências, 2(5), 3-11.

Agra, C. (1997). Droga-crime: A experiência portuguesa: Programa de estudos e resultados. Fundamentação crítica de um programa de estudos e síntese integrativa dos seus resultados. Lisboa: Gabinete de Planeamento e Coordenação do Combate à Droga.

Allen, T. J., Moeller, F. G., Rhoades, H. M., \& Cherek, D. R. (1997). Subjects with a history of drug dependence are more aggressive than subjects with no drug use history. Drug Alcohol Depend, 46(1/2), 95-103.

Barkin, S. L., Smith, K. S., \& Durant, R. H. (2002). Social skills and attitudes associated with substance use behaviors among young adolescents. Journal of Adolescent Health, 30(6), 448-454.

Brook, J. S., Whiteman, M., Balka, E. B., \& Cohen, P. (1997). Drug use and delinquency: Shared and unshared risk factors in African American and Puerto Rican adolescents. Journal Genetic Psychology, 158(1), 25-39.

Correa, L. H. G. (2003). Avaliação do repertório de habilidades sociais em dependentes alcoólicos. Monografia de Conclusão de Curso, Curso de Psicologia, Universidade Federal do Sergipe, Aracaju.

Del Prette, Z. A. P., \& Del Prette, A. (2001). Inventário de habilidades sociais (IHS-Del-Prette): Manual de aplicação, apuração e interpretação. São Paulo: Casa do Psicólogo.

Del Prette, Z. A. P., \& Del Prette, A. (2005). Psicologia das habilidades sociais: Terapia, educação e trabalho. Petrópolis: Vozes.

Dias, C. A. (1982). As teorias e as forças. Psicologia: Revista da Associação Portuguesa de Psicologia, 3, 11-70.

Egger, G. J., Webb, R. A. J., \& Reynolds, I. (1978). Early adolescent antecedents of narcotic abuse. International Journal Addictions, 13(5), 773-781.

Farrel, A. D., Meyer A. L., \& White, K. S. (2001). Beliefs about Aggression and Alternatives Scale (BAAS). Recuperado em 17 nov. 2006, em http:/www.cdc. gov/ncipc/pub-res/measure.html
Ferros, L. (2003). Jovens, drogas e famílias: Uma breve revisão da literatura. Toxicodependências, 9(2), 71-83.

Formiga, N. S., \& Gouveia, V. V. (2003). Adaptação e validação da escala de condutas anti-sociais e delitivas ao contexto brasileiro. Psico (Porto Alegre), 34(2), 367-388.

Gaffney, L. R., Thorpe, K., Young, R., Collet, R., \& Occhipinti, S. (1998). Social skills, expectancies, and drinking in adolescents. Addictive Behaviors, 23(5), 587-599.

Hidalgo, C. H., \& Abarca, N. M. (1992). Comunicacíon interpersonal: Programa de entrenamiento en habilidades sociales. Santiago do Chile: Editorial Universitaria.

Instituto da Droga e da Toxicodependência (IDT). Dependência, opção ou doença? Produção: Plasma. Recuperado em 12 jan. 2007, em http:// www.idt.pt/id.asp?id=p9p25p320

Loeber, R. (1990). Development and risk factors of juvenile antisocial behavior and delinquency. Clinical Psychology Review, 10(1), 1-41.

Loeber, Farrington, Southamer-Lober, \& Van Kammen (1998). "Attitude Toward Delinquency Scale" (ADS). Recuperado em 17 nov. 2006, em http:/www.cdc. gov/ncipc/pub-res/measure.html

Lopes, R. T. (2003). Avaliação do repertório de habilidades sociais em uma amostra randômica de dependentes químicos através do IHS: Del Prette. Monografia de Conclusão de Curso, Curso de Psicologia, Universidade Federal de Juiz de Fora, Juiz de Fora.

Lopes, P., \& Faria, M. (2005). A homofobia em toxicodependentes: Conflitos com o papel de género, atributos pessoais e atitudes perante as mulheres. Toxicodependências, 11(1), 21-35.

Miguel, N. (1997). Toxicodependência: Uma Perspectiva. Toxicodependências, 3(1), 25-30.

Moffitt, T. E. (1993). Adolescence-limited and lifecourse persistent antisocial behavior: A developmental taxonomy. Psychological Review, 100(4), 674-701.

Molina, A. G.-P., \& Gomes, L. F. (1997). Criminologia: Introdução a seus fundamentos teóricos. São Paulo: Revista dos Tribunais. 
Morel, A., Hervé, F., \& Fontaine, B. (1998). Cuidados ao toxicodependente. Lisboa: Climepsi.

Muntaner, C., Walter, D., Nagoshi, C., Fishbein, D., Haertzen, C. A., \& Jaffe, J. H. (1990). Self-report vs. laboratory measures of aggression as predictors of substance abuse. Drug Alcohol Depend, 25(1), 1-11.

Negreiros, J. (1998). Relação entre o consumo de álcool e drogas e comportamentos anti-sociais nos jovens. Toxicodependências, 4(1), 51-58.

Patrício, L. D. (1996). Drogas de vida, vidas de droga. Lisboa: Bertrand.

Quintas, J. (2000). Consumos de drogas: realidades e representações sociais. Toxicodependências, 6(3), $29-42$.

Rubio, J. M. L., \& Anzano, S. M. (1998). Aproximación conceptual a las habilidades sociales. In F. Gil \& J. M. L. Rubio (Orgs.). Habilidades sociales: Teoría, investigación e intervención (pp. 13-23). Madrid: Síntesis.

Seisdedos, N. C. (1988). Cuestionario A-D de conductas antisociais-delictivas. Madrid: TEA.

Spence,J.T., \& Helmreich, R. (1972). The Attitudes toward Women Scale: An objective instrument to measure attitudes toward the rights and roles of women in contemporary society. Journal Supplement Abstract Service Catalog of Selected Documents in Psychology, 2, 66-67.

Spence, J. T., \& Helmreich, R. (1978). Masculinity \& Femininity: Their psychological dimensions, correlates, and antecedents. Austin: University of Texas Press.

Recebido: 10/11/2010

Received: 11/10/2010

Aprovado: 18/04/2011

Approved: 04/18/2011 